\title{
Genetic dissection of drought tolerance in chickpea (Cicer arietinum $\mathbf{L}$. )
}

\author{
Rajeev K. Varshney $\cdot$ Mahendar Thudi $\cdot$ Spurthi N. Nayak $\cdot$ Pooran M. Gaur $\cdot$ Junichi Kashiwagi \\ Lakshmanan Krishnamurthy • Deepa Jaganathan · Jahnavi Koppolu • Abhishek Bohra • Shailesh Tripathi • \\ Abhishek Rathore • Aravind K. Jukanti • Veera Jayalakshmi • Anilkumar Vemula • S. J. Singh • Mohammad Yasin • \\ M. S. Sheshshayee $\cdot$ K. P. Viswanatha
}

Received: 6 August 2013 / Accepted: 31 October 2013 / Published online: 11 December 2013

(C) The Author(s) 2013. This article is published with open access at Springerlink.com

\begin{abstract}
Key message Analysis of phenotypic data for 20 drought tolerance traits in 1-7 seasons at 1-5 locations together with genetic mapping data for two mapping populations provided 9 QTL clusters of which one present on CaLG04 has a high potential to enhance drought tolerance in chickpea improvement.

Abstract Chickpea (Cicer arietinum L.) is the second most important grain legume cultivated by resource poor farmers in the arid and semi-arid regions of the world. Drought is one of the major constraints leading up to $50 \%$ production losses in chickpea. In order to dissect the complex nature of drought tolerance and to use genomics tools for enhancing yield of chickpea under drought conditions, two mapping populations-ICCRIL03 (ICC $4958 \times$ ICC
\end{abstract}

Communicated by A. E. Melchinger.

Electronic supplementary material The online version of this article (doi:10.1007/s00122-013-2230-6) contains supplementary material, which is available to authorized users.

R. K. Varshney $(\bowtie) \cdot$ M. Thudi $\cdot$ S. N. Nayak $\cdot$ P. M. Gaur .

L. Krishnamurthy · D. Jaganathan · J. Koppolu · A. Bohra ·

S. Tripathi · A. Rathore - A. K. Jukanti · A. Vemula

International Crops Research Institute for the Semi-Arid Tropics

(ICRISAT), Hyderabad, India

e-mail: r.k.varshney@ cgiar.org

R. K. Varshney

CGIAR Generation Challenge Programme, c/o CIMMYT,

Mexico, DF, Mexico

Present Address:

S. N. Nayak

University of Florida, Florida, USA

J. Kashiwagi

Hokkaido University, Sapporo, Japan
1882) and ICCRIL04 (ICC $283 \times$ ICC 8261) segregating for drought tolerance-related root traits were phenotyped for a total of 20 drought component traits in 1-7 seasons at 1-5 locations in India. Individual genetic maps comprising 241 loci and 168 loci for ICCRIL03 and ICCRIL04, respectively, and a consensus genetic map comprising 352 loci were constructed (http://cmap.icrisat.ac.in/cmap/sm/ $\mathrm{cp} / \mathrm{varshney/).} \mathrm{Analysis} \mathrm{of} \mathrm{extensive} \mathrm{genotypic} \mathrm{and} \mathrm{pre-}$ cise phenotypic data revealed 45 robust main-effect QTLs (M-QTLs) explaining up to $58.20 \%$ phenotypic variation and 973 epistatic QTLs (E-QTLs) explaining up to $92.19 \%$ phenotypic variation for several target traits. Nine QTL clusters containing QTLs for several drought tolerance traits have been identified that can be targeted for molecular breeding. Among these clusters, one cluster harboring $48 \%$ robust M-QTLs for 12 traits and explaining about $58.20 \%$ phenotypic variation present on CaLG04 has been referred as "QTL-hotspot". This genomic region contains seven SSR markers (ICCM0249, NCPGR127, TAA170,

Present Address:

J. Koppolu

Leibniz Institute of Plant Genetics and Crop Plant Research

(IPK), Gatersleben, Germany

Present Address:

A. Bohra

Indian Institute of Pulses Research (IIPR), Kanpur, India

Present Address:

S. Tripathi

Indian Agricultural Research Institute (IARI), New Delhi, India

Present Address:

A. K. Jukanti

Central Arid Zone Research Institute (CAZRI),

Jodhpur, India 
NCPGR21, TR11, GA24 and STMS11). Introgression of this region into elite cultivars is expected to enhance drought tolerance in chickpea.

\section{Introduction}

Climate change is a global phenomenon that has started to have adverse impact on agriculture. The global temperature is predicted to rise by 2.5 to $4.3{ }^{\circ} \mathrm{C}$ by the end of the century (IPCC 2007). The situation is further likely to be exacerbated by the occurrence of increase in the irregularity of rainfall, drought, flood and land degradation. Higher temperatures, more hot days and heat waves are very likely to hit over nearly all land areas. In this context, drought remains as a big challenge while addressing the problem of food insecurity, hunger and malnutrition especially in the areas where people mainly depend on subsistence farming as a major source of their livelihood (Tuberosa 2012).

Chickpea (Cicer arietinum L.) is grown on low input marginal lands and represents an important component of the subsistence farming. It is the second most important grain legume globally cultivated on an area of 13.20 million hectare (Mha) with an annual production of 11.62 million tons (Mt; FAOSTAT 2011). The global demand for chickpea in 2020 is projected to be $17.0 \mathrm{Mt}$ (up from the current 8.6 Mt; Abate et al. 2012). It is mostly grown on residual moisture from monsoon rains on the Indian subcontinent and semi-arid regions of Sub-Saharan Africa (SSA). India is the largest producer and consumer of chickpea. Among various kinds of abiotic (salinity, heat) stresses affecting the chickpea production, drought stress particularly at the end of the growing season is a major constraint to chickpea production and yield stability in arid and semiarid regions of the world (see Krishnamurthy et al. 2010). Drought causes substantial annual yield losses up to $50 \%$ in chickpea and the productivity remained constant for the past six decades (Ahmad et al. 2005; see Varshney et al. 2010). With predicted climate change scenarios and continuous population explosion, there is a great need to develop high-yielding chickpea varieties with improved drought tolerance (Krishnamurthy et al. 2013a).

\author{
V. Jayalakshmi \\ ANGRAU Regional Agricultural Research Station, \\ Nandyal, India \\ S. J. Singh \\ RAU-Agricultural Research Station, Durgapura, India \\ M. Yasin \\ RAK College of Agriculture, Sehore, India
}

M. S. Sheshshayee $\cdot$ K. P. Viswanatha

University of Agricultural Sciences - Bangalore, Bangalore, India
Drought tolerance is a generic term for a highly complex phenomenon of plant responses. In a practical sense, it is the relative ability of the crop to sustain adequate biomass production and maximize crop yield under increasing water deficit throughout the growing season, rather than the physiological aptitude of the plant for its survival (Serraj and Sinclair 2002). In such context, tolerance to drought is a complex trait with quantitative nature and the underlying mechanism may be due to drought escape, avoidance and tolerance in many crops. Chickpea yields are highly prone to large genotype by environment $(G \times E)$ interactions in marginal environments (Kashiwagi et al. 2008). Breeding for yield under drought conditions using conventional approaches has not been quite successful over the years due to this instability and the poor heritability. Under such circumstances, molecular breeding seems to be a better strategy that can be deployed by targeting drought tolerance component traits with the help of molecular markers.

Understanding genetic basis and identification of molecular markers for drought tolerance component traits are prerequisites for deploying molecular breeding for developing superior genotypes of chickpea. Very recently, significant progress has been made in developing molecular markers and genetic maps in chickpea (Nayak et al. 2010; Gujaria et al. 2011; Gaur et al. 2011; Thudi et al. 2011; Hiremath et al. 2012). While several mapping studies have targeted biotic stress tolerance loci (see Millàn et al. 2006), drought tolerance trait has not yet been targeted systematically for molecular mapping in chickpea. Precision of molecular mapping of a trait, however, is a direct function of precise phenotyping of the trait (Tuberosa 2012; Mir et al. 2012). In the context of drought tolerance, the structure and function of the root system is expected to directly contribute to the transpiration while that of the shoot system structure and function to the transpiration efficiency (TE). Despite their importance in drought tolerance, the roots have attracted little attention in genetic studies mainly because of hard work and skills required for phenotyping root traits (Varshney et al. 2011). As a result of hard work for several years, semi-automated and high-throughput phenotyping techniques for root traits were established at ICRISAT to assess the genetic variability for the root traits in the germplasm collection of chickpea (Kashiwagi et al. 2007). As a result of such endeavors, root traits such as root depth, root biomass and root length density (RLD) were identified as most promising traits in chickpea for terminal drought tolerance, as these help in greater extraction of soil moisture (Kashiwagi et al. 2006; Varshney et al. 2011). Importance of such root traits contributing to drought tolerance has also been demonstrated in some other legumes (Wang et al. 2004) and cereals (Toorchi et al. 2004; Tuberosa and Salvi 2007).

In addition to the root traits, another important trait for drought tolerance is water-use efficiency (WUE) or TE 
(Passioura 1977; Kashiwagi et al. 2013). Carbon isotope discrimination $\left(\delta^{13} \mathrm{C}\right)$ is considered the best method to screen germplasm for WUE. While a range of reports are available on correlation between $\delta^{13} \mathrm{C}$ and TE, a positive correlation was found between $\delta^{13} \mathrm{C}$ and TE under drought stress environments in chickpea (Kashiwagi et al. 2006). Furthermore, irrespective of root traits or TE, yield and yield component traits and harvest index (HI) are always considered the most reliable traits for breeding for drought tolerance.

With an objective to dissect drought tolerance into component traits and understand genetic basis and identify molecular markers for different component traits, this study undertakes extensive phenotyping and genotyping and their comprehensive analysis on two intra-specific recombinant inbred line (RIL) populations. This study is the first report on the development of the most-dense genetic maps on intra-specific populations and identification of both main-effect QTLs (M-QTLs) as well as epistatic QTLs (E-QTLs) for different drought tolerance traits in chickpea. Most importantly, this study reports a "QTL-hotspot" in the chickpea genome, identified in analysis on both RIL populations, that contain $45 \mathrm{M}-\mathrm{QTL}$ and $973 \mathrm{E}-\mathrm{QTLs}$ for several drought tolerance traits contributing up to $58.20 \%$ phenotypic variation for targeted traits. In summary, this study provides molecular markers for deploying molecular breeding for drought tolerance, a very complex trait, to develop superior chickpea varieties.

\section{Materials and methods}

\section{Plant material and DNA extraction}

Based on screening of mini-core collection for drought tolerance-related root traits, ICC 4958 (a drought tolerant breeding line developed by Jawaharlal Nehru Krishi Vishwa Vidyalaya, Jabalpur, Madhya Pradesh, India) and ICC 8261 (a drought tolerant landrace from Lebanon) assembled in ICRISAT's genebank in 1973 and 1974 were found to possess larger root system, while ICC 283 and ICC 1882 are landraces collected from India and assembled in ICRISAT's genebank in 1974 and 1973, respectively, were found to possess shorter root system. These phenotypically and genetically distinct genotypes were used for developing two intra-specific mapping populations, namely ICCRIL03 (264 RILs from ICC $4958 \times$ ICC 1882) and ICCRIL04 (288 RILs from ICC $283 \times$ ICC 8261), at ICRISAT.

DNA from parental genotypes as well as from 232 and 234 RILs of ICCRIL03 and ICCRIL04, respectively, was isolated employing high-throughput mini-DNA extraction method as mentioned in Cuc et al. (2008).
Phenotypic evaluation

The above-mentioned populations (ICCRIL03 comprising 264 RILs and ICCRIL04 comprising 288 RILs) were evaluated for a total of 20 drought tolerance traits including 6 root traits, 6 yield and yield-related traits, 5 morphological traits, 2 phenological traits and 1 physiological trait in three replications in 1-7 seasons (2005-2006, 20062007, 2007-2008, 2008-2009, 2009-2010, 2010-2011 and 2011-2012) at 1-5 locations in India, namely Patancheru (PAT), Nandyal (NDL), Durgapura (DUG), Hiriyur (HIR) and Sehore (SEH) (ESM Table S1).

\section{Root trait phenotyping under rainout shelter (ROS) conditions}

Both populations were phenotyped for root traits such as root length (RL, cm), root length density $\left(\mathrm{RLD}, \mathrm{cm} \mathrm{cm}^{-3}\right.$ ), root dry weight (RDW, g), rooting depth $(\mathrm{RDp}, \mathrm{cm})$, root surface area $\left(\mathrm{RSA}, \mathrm{cm}^{2}\right)$, root volume $\left(\mathrm{RV}, \mathrm{cm}^{3}\right)$, ratio between RDW and total plant dry weight (RTR, \%), and one morphological trait, shoot dry weight (SDW, g) in cylinder culture in three replications in rainout shelter using semi-automated high-throughput precise phenotyping facility at ICRISAT, Patancheru as described earlier (Kashiwagi et al. 2006). The ICCRIL03 was phenotyped during post-rainy season of 2005 and 2007, while ICCRIL04 was phenotyped during post-rainy season of 2006 and 2010.

\section{Morphological, phenological and yield-related traits under field conditions}

ICCRIL03 and ICCRIL04 were phenotyped for five morphological traits (plant height, PHT, cm; plant stand, PS; plant width, PWD, cm; primary branches, PBS; secondary branches, SBS), two phenological traits (days to $50 \%$ flowering, DF; days to maturity, DM) and six yield-related traits (100-seed weight, 100-SDW, g; pods per plant, POD; seeds per pod, SPD; Yield, YLD, g; biomass, BM, g; harvest index, HI, \%) during post-rainy 2005-2006, 20062007, and 2007-2008 seasons under rainfed (RF) environments at PAT. In addition, ICCRIL03 was also phenotyped under rainfed condition during post rainy 2008 at PAT, SEH, DUG and NDL (ESM Table S1).

Furthermore, both populations were phenotyped for the above-mentioned morphological, phenological and yieldrelated traits under RF and irrigated (IR) environments. ICCRIL03 was phenotyped during post-rainy 2009-2010 at five locations (PAT, NDL, DUG, HIR and SEH), while ICCRIL04 was phenotyped during post-rainy 2010-2011 and 2011-2012 at four locations (PAT, NDL, DUG and SEH). 


\section{Phenotyping for physiological trait}

Delta carbon ratio $\left(\delta^{13} \mathrm{C}\right)$ is considered as an indirect measure of TE, which is an important measure of drought tolerance. For estimating the $\delta^{13} \mathrm{C}$, fourth and fifth fully expanded leaves from top of the stems of ICCRIL03 population were collected during post-rainy season 2008-2009 at four locations PAT, DUG, NDL and SEH as mentioned in Kashiwagi et al. (2006).

\section{Analysis of variance, correlations and heritability}

The analysis of variance (ANOVA) for all traits was computed considering genotypes as random effect. Best Linear Unbiased Predictors (BLUPs) were estimated by using SAS MIXED procedure (SAS Inst. 2002-2008, SAS V9.2). In addition, the least square means (LSM; genotype as fixed effect), standard error of differences (SED), least significant difference (LSD) and descriptive statistics such as coefficient of determination $\left(R^{2}\right)$, coefficient of variation (CV) and grand mean were determined for all the traits studied. Genotypic and phenotypic variance components were also estimated to calculate broad sense heritability $\left(H^{2}\right)$. Drought tolerance index (DTI) and drought susceptibility index (DSI) were computed as mentioned in Golabadi et al. (2006).

\section{PCR and marker analysis}

A total of 2,717 markers including 2,410 simple sequence repeats (SSRs) (311 SSRs from Nayak et al. 2010; 1,344 SSRs from Thudi et al. 2011; 241 SSRs from Winter et al. 1999; 233 SSRs from Lichtenzveig et al. 2005; 181 SSRs from Gaur et al. 2011; 100 SSRs from Sethy et al. 2006), 230 genic molecular markers (GMMs) from Gujaria et al. (2011) and 77 EST-SSRs from Varshney et al. (2009) were screened on the parental lines of two mapping populations.

Polymorphic markers (321 for ICCRIL03 and 230 for ICCRIL04) were used for genotyping respective mapping populations (ESM Table S2). PCR analysis for all SSR markers were performed in $5 \mu$ l reaction volume employing GeneAmp ${ }^{\circledR}$ PCR System 9700 DNA thermal cycler (Applied Biosystems, USA). Marker genotyping for ICCM and CaM series SSRs on RILs was done as mentioned in our earlier studies (Nayak et al. 2010; Thudi et al. 2011). Similarly, genotyping for GMMs and diversity arrays technology (DArT) loci was done on the RILs in the same way as mentioned in our earlier studies (Gujaria et al. 2011; Thudi et al. 2011).
Construction of genetic maps and consensus maps

Genotyping data were assembled for all segregating makers (ESM Table S2) on 232 and 234 RILs of ICCRIL03 and ICCRIL04 mapping populations, respectively, and linkagebased mapping was performed using JoinMap version 4.0 (Van Ooijen 2006) as described in Bohra et al. (2012). A consensus genetic map was derived from two intra-specific mapping populations using software JoinMap 4.0 as described in Bohra et al. (2012).

\section{QTL analysis}

Candidate QTL regions for drought tolerance were identified using two trait mapping approaches: (1) interval mapping for identifying M-QTLs and (2) epistatic interaction analysis for detecting QTL interactions. Composite interval mapping (CIM) was employed for detection of M-QTLs using Windows QTL Cartographer version 2.5 (Wang et al. 2010). In parallel, for detection of E-QTLs, a two-locus QTL analysis or two-dimensional (2D) genome scanning was conducted using software QTLNetwork version 2.0 (http://ibi.zju.edu.cn/software/qtlnetwork/) allowing simultaneous detection of M-QTLs, E-QTLs, and the QTLs involved in epistatic $(Q \times Q)$ and QTL by environment $(Q \times E)$ interactions as described in Gautami et al. (2012). The threshold for declaring QTL is set to $P$ value of 0.05 by permutation method (1,000 permutations).

\section{Results}

Phenotypic trait variation and heritability

The two intra-specific mapping populations ICCRIL03 and ICCRIL04 were phenotyped for a total of 20 drought component traits in 1-7 seasons at 1-5 locations in India. The component traits, their codes and units of measurement, locations, seasons and environments have been listed in Table 1. In addition, DTI and DSI were computed based on phenotypic data from both RF and IR environments. The key features of extensive phenotyping data are given below and detailed analysis such as mean performance, range of trait values, and $\mathrm{H}^{2}$ of traits at different locations, environments and seasons on both RILs are provided in ESM Tables S3 and S4.

\section{Root traits}

As roots are the first part of the plants exposed to drought stress, six root traits, namely RLD, RDW, RDp, RV, RSA, 
Table 1 Traits, trait codes, units, locations of phenotyping and environments and mapping populations

\begin{tabular}{|c|c|c|c|}
\hline Names & Code (units) & Names & Code (units) \\
\hline \multicolumn{2}{|l|}{ Root traits } & \multicolumn{2}{|l|}{ Drought indices } \\
\hline Root length density & $\operatorname{RLD}\left(\mathrm{cm} \mathrm{cm}^{-3}\right)$ & Drought tolerance index & DTI \\
\hline Root dry weight & RDW (g) & Drought susceptibility index & DSI \\
\hline Rooting depth & $\mathrm{RDp}(\mathrm{cm})$ & Locations & \\
\hline Root surface area & $\operatorname{RSA}\left(\mathrm{cm}^{2}\right)$ & Patancheru & PAT \\
\hline Root volume & $\mathrm{RV}\left(\mathrm{cm}^{3}\right)$ & Nandyal & NDL \\
\hline $\begin{array}{l}\text { Root dry weight/total plant dry } \\
\text { weight ratio }\end{array}$ & RTR (\%) & Sehore & SEH \\
\hline \multicolumn{2}{|l|}{ Morphological traits } & Durgapura & DUG \\
\hline Shoot dry weight & SDW (g) & Hiriyur & HIR \\
\hline Plant height & PHT (cm) & Environments & \\
\hline Plant width & PWD (cm) & Rainfed & $\mathrm{RF}$ \\
\hline Primary branches & PBS & Irrigated & IR \\
\hline Secondary branches & SBS & Cylinder culture & $\mathrm{CC}$ \\
\hline \multicolumn{2}{|l|}{ Phenological traits } & \multicolumn{2}{|l|}{ Seasons } \\
\hline Days to $50 \%$ flowering & DF & $2005-06$ & 2005 \\
\hline Days to maturity & $\mathrm{DM}$ & $2006-07$ & 2006 \\
\hline \multicolumn{2}{|l|}{ Yield and yield-related traits } & $2007-08$ & 2007 \\
\hline Pods/plant & POD & 2008-09 & 2008 \\
\hline Seeds/pod & SPD & 2009-10 & 2009 \\
\hline 100-seed weight & 100SDW (g) & $2010-11$ & 2010 \\
\hline Biomass & $\mathrm{BM}(\mathrm{g})$ & $2011-12$ & 2011 \\
\hline Harvest index & $\mathrm{HI}(\%)$ & Mapping populations & \\
\hline Yield & YLD $(g)$ & ICC $4958 \times$ ICC 1882 & ICCRIL03 \\
\hline \multicolumn{2}{|l|}{ Transpiration efficiency related traits } & ICC $283 \times$ ICC 8261 & ICCRIL04 \\
\hline Delta carbon ratio & $\delta^{13} \mathrm{C}$ & & \\
\hline
\end{tabular}

and RTR, were used for phenotyping of two RIL populations. In ICCRIL03, the genetic variability for RLD among RILs was high in $2007\left(0.1-0.47 \mathrm{~cm} \mathrm{~cm}^{-3}\right)$ compared to $2005\left(0.22-0.46 \mathrm{~cm} \mathrm{~cm}^{-3}\right)$ at 35 days after sowing (DAS) (ESM Table S3). However, $H^{2}$ was high in $2005(0.61)$ compared to 2007 (0.34). The variation among RILs for RDW was high in $2005(0.43-1.18 \mathrm{~g})$ compared to 2007 (0.27-0.89 g); however, the $H^{2}$ was low in both seasons (0.29 in 2005 and 0.21 in 2007). Although genotypic variability for RDp was observed among RILs in both the seasons, no significant difference was observed between parents of each of two mapping populations (ESM Table S3). Further, the $H^{2}$ was very low for RDp compared to any other root traits studied. The genotypic variability among RILs for RV and RSA was significant in 2005 and non-significant in 2007. The variation for RTR was high in 2007 (21.92-50.9\%) compared to 2005 (22.43-39.23\%); however, $H^{2}$ was high in 2005 (0.56) compared to $2007(0.26)$.

In the case of ICCRIL04, at 35 DAS, RLD ranged from 0.20 to $0.45 \mathrm{~cm} \mathrm{~cm}^{-3}$ (2006) and 0.18 to $0.46 \mathrm{~cm} \mathrm{~cm}^{-3}$ (2010). However, the genotypic variability was significant only in 2010 (ESM Table S4). The genotypic variability was highly significant $(<0.001)$ for RDW, RV and RTR in both seasons; however, the $H^{2}$ was moderate for these traits (0.33-0.48).

\section{Morphological traits}

Drought stress affects several morphological traits and therefore two RIL populations were also phenotyped for SDW, PHT, PWD, PBS and SBS.

In the case of ICCRIL03, at 35 DAS, genetic variability for SDW among RILs was high in 2007 (0.53-2.23 g) compared to 2005 (1.11-2.75 g). Further, significant differences $(P<0.0001)$ for SDW among RILs were observed in both seasons in addition to high $H^{2}$. PHT ranged from 21.6 to $62.4 \mathrm{~cm}$ under RF environment in 2008 across four locations PAT, NDL, DUG and SEH (ESM Table S3). Further, genetic variability for PHT was highly significant $(P<0.001)$ under RF environment at PAT, SEH, NDL, and DUG in 2008 with high $H^{2}(0.75-0.99)$. In addition, PHT also differed significantly among RILs in 2009 both at PAT and NDL under RF and IR environments. However, no significant genetic variability for PHT was observed in 2009 under RF and IR environments in case of SEH and DUG (ESM Table S3). In ICCRIL04, at 35 DAS, genetic 
variability for SDW among RILs was high in 2010 (0.75$2.78 \mathrm{~g})$ compared to $2006(0.56-2.10 \mathrm{~g})$. Significant difference for PHT was observed at all locations and all environments in 2010 and 2011 except DUG under RF environment in 2010 (ESM Table S4).

\section{Phenological traits}

Two phenological traits, namely DF and DM, that are important for breeding were recorded on two RIL populations.

In the case of ICCRIL03, phenotyping of these traits showed significant genetic variability for DF under RF environment in 2008 at PAT, SEH and NDL. However, DF did not differ significantly in both RF and IR environments in SEH in 2009. Similarly, in the case of DUG, no significant difference among RILs was noted in IR environment in 2009 (ESM Table S3). For DM, a significant difference among RILs was observed under RF in 2008 at all four locations (PAT, DUG, NDL and SEH) studied. Further, significant differences were also observed for DM under RF and IR environments at PAT, NDL and DUG in 2009. However, in 2009 at SEH under IR environment, there was no significant difference among RILs (ESM Table S3).

In the case of ICCRIL04, DF was significant across all environments and at all locations (PAT, DUG, NDL and SEH) in 2010 in both RF and IR environments. In addition, DF was also significant across five locations (PAT, DUG, NDL, HIR and SEH) under both IR and RF in 2011. Similarly, DM was also significant across all environments (RF and IR) in 2010 and 2011 at all but one location PAT under RF in 2010 (ESM Table S4).

\section{Yield and yield-related traits}

Yield, especially under drought stress, is the ultimate requirement for farmers and breeders. Therefore, two RIL populations were phenotyped for yield and yield-related traits like POD, SPD, BM, 100SDW, HI and YLD under RF and IR conditions. Among yield-related traits in ICCRIL03, no significant variability was observed for POD and SPD except for PAT in 2009 under IR environment. Except three locations (SEH in 2009 under both environments, PAT in 2009 under RF and DUG in 2008 under RF environment), the $H^{2}$ was high in case of 100SDW at all locations in 2008 and 2009. It ( $H^{2}$ value) ranged from 0.64 to 0.99 across locations and environments studied (ESM Table S3). Significant genetic variation for BM was observed among RILs in 2008 under RF at PAT, SEH and NDL. However, in 2009 significant genetic variability for BM was observed only in the case of NDL under both RF and IR environments. In 2008, under RF environment, genetic variability for HI was significant only at PAT and NDL locations with
$H^{2}$ of 0.45 and 0.68 , respectively. Further in the case of 2009, genetic variability for HI under RF and IR was significantly high in two locations, PAT and NDL.

In the case of ICCRIL04, the genetic variability for 100SDW, BM, YLD and HI was significant among RILs at all locations (PAT, DUG, NDL, HIR and SEH), both seasons (2010 and 2011) and both environments (IR and RF) except BM at PAT in 2011 under RF condition (ESM Table S4). $H^{2}$ for 100SDW across locations and environments ranged from 0.87 to 0.99 . Similarly, $H^{2}$ was also high for BM (0.47-0.89), YLD (0.6-0.99) and HI (0.56-0.99).

Analysis of variance and trait correlations

The combined ANOVA revealed significant differences among RILs of both populations (ICCRIL03 and ICCRIL04) for all the above-mentioned traits $(P<0.05$, 0.01 and 0.001; ESM Tables S5 and S6).

In the case of ICCRIL03, significant effect of location was observed for all morphological traits, yield-related traits and block effects were significant for all traits except yield-related traits measured at SEH and DUG location under IR environment. Significant interaction between RILs and location was observed for all traits at $1 \%$ level of significance. The mean square values for 2 years $(2005$ and 2007) differed significantly from each other for all root traits. Highly significant differences $(P<0.001)$ were found in genotypes (RILs) for all traits except for the trait RV.

Correlation is a pragmatic approach to develop selection criteria for accumulating optimum combination of yield contributing traits in a simple genotype. Among root traits, RTR has significant negative correlation with RL, RLD, and RSA and non-significant correlation with RDp in ICCRIL03 across both seasons (2005 and 2007). $\delta^{13} \mathrm{C}$, an indirect measure of plant TE has a significant positive correlation with $\mathrm{HI}$ and a negative correlation with PHT across locations during 2008, while correlations were non-significant in case of $\mathrm{BM}$, YLD with $\delta^{13} \mathrm{C}$ with all other traits. However, in the case of ICCRIL04, RTR has only negative correlation with SDW. In addition, a non-significant negative correlation was observed between RLD and RTR. Nevertheless, all other traits have significant positive correlation in the case of ICCRIL04 (ESM Table S7).

Furthermore, a significant positive correlation was found between YLD and 100SDW, BM and a negative correlation between YLD and DF, DM, as expected across locations and seasons (ESM Table S7) in both RIL populations.

Component genetic maps

Screening of 2,717 SSR markers on the parental lines resulted in identification of 321 and 230 polymorphic 
markers on ICCRIL03 and ICCRIL04, respectively (Lichtenzveig et al. 2005; Sethy et al. 2006; Varshney et al. 2009; Nayak et al. 2010; Gujaria et al. 2011; Gaur et al. 2011; Thudi et al. 2011; ESM Table S2). The genotyping data were generated for the polymorphic markers on respective mapping populations. As a result, 241 marker loci including 214 SSRs, 6 GMMs and 21 DArT loci were placed on to genetic map for ICCRIL03 (Table 2; ESM Figure S1; http://cmap.icrisat.ac.in/cmap/sm/cp/varshney/) and 168 marker loci (151 SSRs, 10 GMMs and 7 DArT loci) in the case of ICCRIL04 (Table 2; ESM Figure S2; http://cmap.icrisat.ac.in/cmap/sm/cp/varshney/). In total, $62(26.95 \%)$ markers in the case of ICCRIL04 and $80(24.92 \%)$ markers in the case of ICCRIL03 remained unmapped. Varying levels of marker densities were recorded for different linkage groups (LGs) in both the maps and the average inter-marker distances were 2.71 and $3.27 \mathrm{cM}$ in the case of ICCRIL03 and ICCRIL04, respectively (Table 2). Of 46 markers with segregation distortion, 9 markers were not mapped in case of ICCRIL03, while in the case of ICCRIL04, 20 markers remained unmapped.

\section{QTLs for drought tolerance component traits}

To understand the genetic and molecular basis of drought tolerance, developed genetic maps and extensive phenotyping data generated on both RIL populations were analyzed in details for identification of both main-effect QTLs as well as the QTLs showing epistatic interactions.

\section{Main-effect QTLs (M-QTLs)}

For both RIL populations, M-QTLs were identified using QTL Cartographer and QTLNetwork programs (ESM Figures S1 and S2). In the case of ICCRIL03, QTL Cartographer identified a total of $77 \mathrm{M}$-QTLs including 36 M-QTLs for yield-related traits; 12 M-QTLs for morphological traits; 11 M-QTLs for root traits; 9 M-QTLs for phenological traits; $7 \mathrm{M}$-QTLs for drought tolerance indices and $2 \mathrm{M}$-QTLs for $\delta^{13} \mathrm{C}$ (ESM Table S8). In case if one of two flanking markers is common in more than one QTL, we have considered that region as only one genomic region that contains $>1$ QTL. By following this criteria, 77 M-QTLs identified were present in 36 genomic regions. On the other hand, QTLNetwork analysis provided 62 M-QTLs in 22 genomic regions. These QTLs include 26 M-QTLs for yield and yield-related traits; 14 M-QTLs for morphological traits; $10 \mathrm{M}$-QTLs for phenological traits; 5 M-QTLs for root traits; 6 M-QTLs for drought tolerance indices and $1 \mathrm{M}-\mathrm{QTL}$ for $\delta^{13} \mathrm{C}$ (ESM Table S9). Of the 77 M-QTL detected by QTL Cartographer, nearly $40 \%$ of the QTLs (30 M-QTLs) were located on CaLG04 followed by CaLG01 (12 M-QTLs). The similar observations

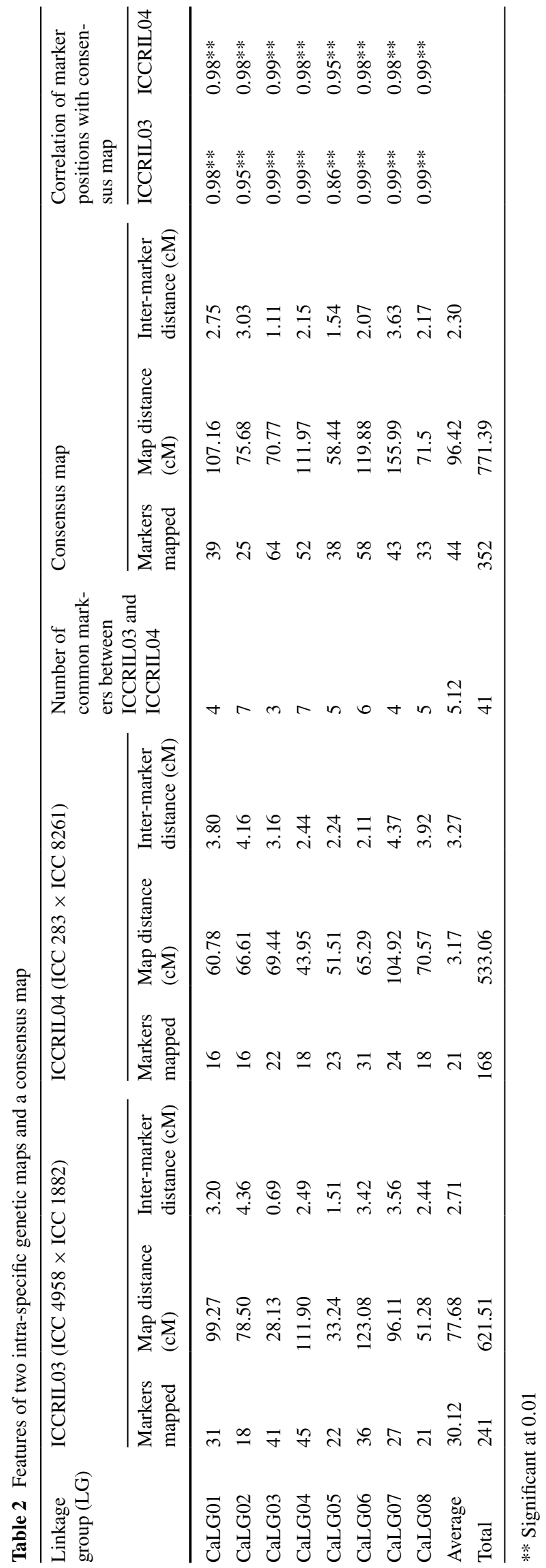


were made in QTLNetwork analysis in which 28 of 62 M-QTLs were present on CaLG04 followed by CaLG01 (14 M-QTLs).

In the case of ICCRIL04, 51 M-QTLs in 25 genomic regions were identified by QTL Cartographer, which include $15 \mathrm{M}$-QTLs for yield-related traits, 14 M-QTLs for phenological traits, 11 M-QTLs for morphological traits, $7 \mathrm{M}$-QTLs for root-related traits and $4 \mathrm{M}$-QTLs for drought indices (ESM Table S8). QTLNetwork detected $13 \mathrm{M}$-QTLs in ten genomic region and includes $5 \mathrm{M}$-QTLs for phenological traits, $4 \mathrm{M}$-QTLs for morphological traits, $3 \mathrm{M}$-QTLs for yield-related traits and $1 \mathrm{M}$-QTL for DTI (ESM Table S9; ESM Figure S2). Majority of QTLs identified by any of these programs were located on CaLG01 followed by CaLG08, CaLG06.

\section{Epistatic QTLs (E-QTLs)}

For understanding the complexity of drought tolerance traits, QTLNetwork and genotype matrix mapping program (GMM program) were used to detect E-QTLs in both RILs. For instance in the case of ICCRIL03, by considering two loci interactions, a total of 26 E-QTLs were identified that include 15 E-QTLs detected by QTLNetwork (ESM Table S10) and 11 detected by GMM program (ESM Table S11). These QTLs contribute up to $26.18 \%$ phenotypic variation for 10 of 20 traits phenotyped and DTI. GMM program also provided 693 E-QTLs by considering three loci interactions for all the 20 traits and both drought indices with 7.09-91.56 \% phenotypic variation (ESM Table S11).

Similarly in the case of ICCRIL04, a total of 13 E-QTLs were detected by QTLNetwork (ESM Table S10) and no QTL was detected by GMM program for two loci interaction (ESM Table S12). These QTLs contribute from 3.57 to $13.25 \%$ phenotypic variation for 7 of 20 traits phenotyped and DTI. GMM program also provided 295 E-QTLs by considering three loci interactions for 16 traits and drought indices with $0.49-92.19 \%$ phenotypic variation (ESM Table S12).

\section{Trait dissection}

Comprehensive QTL analysis of both M-QTLs and E-QTLs provided an opportunity to analyze drought tolerance component traits in depth. As QTL analysis was undertaken on phenotypic data for 20 traits and 2 drought indices, collected in 1-7 years (seasons) at 1-5 locations, phenotypic data collected in a given year at given location were considered as one environment. By considering this criterion, QTL analysis was undertaken for 20 traits across 20 environments. Phenotypic variation explained (PVE) by M-QTLs ranged from 2.34 to $58.20 \%$ in case of ICCRIL03 and 2.95 to $31.32 \%$ in case of ICCRIL04 (ESM Table S8), while E-QTLs explained 0.75 to $91.56 \%$ PVE in ICCRIL03 and 0.49 to $92.19 \%$ PVE in the case of ICCRIL04 (ESM Tables S11 and S12). For trait dissection in comprehensive manner, only robust M-QTLs and E-QTLs that contribute $>10 \%$ PVE were considered into account. If the QTL for a given trait appeared in more than one location, it was considered as 'stable' QTL and if this appears in more than 1 year/season, the QTL was considered as 'consistent' QTL.

Furthermore, a quick comparison of M-QTLs identified by QTL Cartographer and QTLNetwork showed that M-QTLs detected by QTL Cartographer include all or key QTLs detected by QTLNetwork; therefore, M-QTLs identified by QTL Cartographer only were considered for trait dissection analysis. Similarly, GMM program analysis provided more comprehensive E-QTLs (both two loci as well as three loci interactions) as compared to QTLNetwork, and thus GMM program analysis-based E-QTLs with 3 loci interactions were included for trait dissection analysis.

In brief, M-QTLs detected by QTL Cartographer with $>10 \%$ PVE and E-QTLs (3 loci interactions) detected by GMM program with $>10 \%$ PVE were used for comprehensive genetic analysis of drought tolerance component traits.

\section{Root traits}

Of the six root traits analyzed, robust M-QTLs were identified for three traits one each for RLD, RSA and RTR in ICCRIL03 (Table 3) and therefore no stable and consistent QTL was detected. In terms of robust E-QTLs, robust 3 loci epistatic interactions were observed for all six traits (Table 4). For instance, for RLD, one E-QTL [TA127 (BB) TA180 (BB) ICCM0065 (BB)] contributing $31.41 \%$ PVE was observed in 2005. In 2007, although eight robust E-QTLs contributing from 23.5 to $33.23 \%$ PVE were identified, one locus, namely TA180, was common in robust E-QTLs of 2005 as well as in all eight robust E-QTLs of 2007 (ESM Table S11).

In the case of ICCRIL04, although no robust M-QTL was identified for any trait, 3-11 robust E-QTLs with up to $44.61 \%$ PVE were identified for RLD, RDW, RTR and RV traits (Table 4). In majority of the cases, identified robust E-QTLs were consistent, as at least one locus present in a robust E-QTL identified in 1 year was also present in the robust E-QTL identified in the other year.

\section{Morphological traits}

Of the five traits analyzed for morphological characters, five robust M-QTLs (4 for PHT and 1 for SDW) with 
Table 3 Main-effect QTLs (M-QTLs) for drought tolerance related traits identified in two RIL populations

\begin{tabular}{|c|c|c|c|c|c|c|c|c|}
\hline \multirow[t]{2}{*}{ Trait } & \multicolumn{3}{|c|}{ ICCRIL03 (ICC 4958 × ICC 1882) } & \multirow{2}{*}{$\begin{array}{l}\text { Phenotypic variation } \\
\text { explained (PVE, \%) }\end{array}$} & \multicolumn{3}{|c|}{ ICCRIL04 (ICC $283 \times$ ICC 2861) } & \multirow{2}{*}{$\begin{array}{l}\text { Phenotypic variation } \\
\text { explained (PVE, \%) }\end{array}$} \\
\hline & $\begin{array}{l}\text { No. of } \\
\text { QTLs }\end{array}$ & $\begin{array}{l}\text { Stable } \\
\text { QTLs }\end{array}$ & $\begin{array}{l}\text { Consistent } \\
\text { QTLs }\end{array}$ & & No. of QTLs & $\begin{array}{l}\text { Stable } \\
\text { QTLs }\end{array}$ & $\begin{array}{l}\text { Consistent } \\
\text { QTLs }\end{array}$ & \\
\hline \multicolumn{9}{|l|}{ Root } \\
\hline RLD & 1 & - & - & 10.90 & - & - & - & - \\
\hline RSA & 1 & - & - & 10.26 & - & - & - & - \\
\hline RTR & 1 & - & - & 16.67 & - & - & - & - \\
\hline \multicolumn{9}{|c|}{ Morphological } \\
\hline SDW & 1 & - & 1 & $13.89-17.59$ & - & - & - & - \\
\hline PHT & 4 & 1 & 2 & $10.00-30.20$ & 2 & - & 1 & $11.27-31.32$ \\
\hline PWD & - & - & - & - & 1 & - & - & 15.84 \\
\hline \multicolumn{9}{|c|}{ Phenological } \\
\hline DF & 2 & 1 & 1 & $10.51-26.87$ & 4 & 1 & 2 & $10.66-18.97$ \\
\hline DM & 3 & 1 & 1 & $12.13-19.71$ & 4 & 1 & - & $10.47-16.79$ \\
\hline \multicolumn{9}{|c|}{ Yield related } \\
\hline 100SDW & 2 & 1 & 1 & $10.31-58.20$ & 1 & - & 1 & $17.14-26.67$ \\
\hline $\mathrm{BM}$ & 2 & - & - & $10.95-21.32$ & - & - & - & - \\
\hline $\mathrm{HI}$ & 3 & - & - & $10.67-14.36$ & 2 & - & - & $12.06-14.04$ \\
\hline POD & 1 & - & 1 & $10.19-23.18$ & 1 & - & 1 & $12.13-14.37$ \\
\hline SPD & 1 & - & - & 42.07 & - & - & - & - \\
\hline YLD & 2 & - & - & $13.98-15.71$ & 3 & - & - & $10.06-18.55$ \\
\hline \multicolumn{9}{|c|}{ Drought indices } \\
\hline DTI & 1 & - & - & 11.23 & 2 & - & - & $11.27-12.12$ \\
\hline Total & 25 & 4 & 7 & & 20 & 2 & 5 & \\
\hline
\end{tabular}

up to $30.20 \%$ PVE were identified for two traits (SDW and PHT) in ICCRIL03 (Table 3). Of these five robust M-QTLs, a QTL named ' $Q R 3 s d w 01$ ' flanked by 'TAA170NCPGR21' on CaLG04 appeared consistently for two seasons (2005 and 2007) for SDW. Further, two QTLs for PHT ('QR3pht01' flanked by 'CaM1760-CaM0399' on CaLG06 and 'QR3pht03' flanked by 'NCPGR127-NCPGR21' on CaLG04) were consistent in 2005, 2007, 2008 and 2009. In addition, the QTL 'QR3pht03' was stable across 1-5 locations (PAT, SEH, NDL, DUG and HIR) (ESM Table S8). In terms of epistatic interactions, 71 robust E-QTLs with up to $76.54 \%$ PVE were detected for all 5 traits analyzed (Table 4; ESM Table S11). Majority of these QTLs have at least one common locus interacting with other two loci (ESM Table S11).

In the case of ICCRIL04, three robust M-QTLs (2 for PHT and 1 for PWD) with up to $31.32 \%$ PVE were detected for PHT and PWD. Of these three robust M-QTLs, one QTL 'QR4pht02' flanked by 'CaM0772-TS45' on CaLG08 consistently appeared in two seasons (2005 and 2006). Furthermore, 41 robust E-QTLs with up to $76.26 \%$ PVE were detected for all five traits (Table 4). Interestingly, one locus 'TA127' was observed in 11 of 31 robust E-QTLs identified for PHT (ESM Table S12).

\section{Phenological traits}

A total of five robust M-QTLs (3 M-QTL for DM and 2 M-QTL for DF) with up to $26.87 \%$ PVE were detected for DF and DM in the ICCRIL03. Of these five M-QTLs, in case of DF, 'QR3df01' QTL flanked by 'NCPGR164CaM1918' on CaLG08 was consistent in three seasons (2005, 2008 and 2009) and stable at four locations (PAT, HIR, NDL and DUG). While, in the case of DM, the QR3dm01 flanked by 'NCPGR164-CaM1918' on CaLG08 was consistent for two seasons (2008 and 2009) and stable at three locations (PAT, HIR and DUG) (Table 3; ESM Table S8). In addition, although a large number of robust E-QTLs (220) were detected for all traits studied, one locus, namely 'NCPGR203', had the highest interaction in 21 E-QTLs for DM (ESM Table S11).

In the case of ICCRIL04, eight robust M-QTLs (4 each for DF and DM) were identified with up to $18.97 \%$ PVE. In case of DF, one QTL 'QR4df01' flanked by 'CaM1753cpPb-677529' on CaLG03 was consistent across two seasons (2005 and 2006) and another QTL 'QR4df06' flanked by 'TA103II-TA122' was consistent across two seasons (2010 and 2011) and stable across two locations (HIR and $\mathrm{NDL}$ ). In the case of DM, one QTL ' $Q R 4 d m 05$ ' flanked by 
Table 4 Summary on three loci epistatic interactions in two RIL populations based on genotype matrix mapping program (GMM program) analysis
${ }^{\text {a }} P V E$ phenotypic variation explained

\begin{tabular}{|c|c|c|c|c|}
\hline \multirow[t]{3}{*}{ Traits } & \multicolumn{2}{|l|}{ ICCRIL03 } & \multicolumn{2}{|l|}{ ICCRIL04 } \\
\hline & \multicolumn{2}{|c|}{ Three loci interactions } & \multicolumn{2}{|c|}{ Three loci interactions } \\
\hline & No. of QTLs & $\operatorname{PVE}^{\mathrm{a}}(\%)$ & No. of QTLs & $\operatorname{PVE}^{\mathrm{a}}(\%)$ \\
\hline \multicolumn{5}{|l|}{ Root } \\
\hline RLD & 9 & $23.49-33.23$ & 9 & $13.25-44.20$ \\
\hline RDW & 2 & $17.77-20.72$ & 11 & $18.03-44.61$ \\
\hline $\mathrm{RDp}$ & 12 & $10.71-24.39$ & - & - \\
\hline RSA & 11 & $14.93-42.97$ & - & - \\
\hline RTR & 4 & $23.25-34.99$ & 4 & $17.82-22.60$ \\
\hline $\mathrm{RV}$ & 16 & $16.61-19.53$ & 3 & $29.37-36.61$ \\
\hline \multicolumn{5}{|c|}{ Morphological } \\
\hline SDW & 11 & $12.80-21.76$ & 3 & $21.12-76.26$ \\
\hline PHT & 39 & $14.36-76.54$ & 31 & $14.63-69.50$ \\
\hline PWD & 3 & $23.35-37.28$ & 3 & $13.46-16.06$ \\
\hline PBS & 13 & $12.70-28.45$ & 1 & 32.53 \\
\hline SBS & 5 & $13.26-27.93$ & 3 & $36.92-44.41$ \\
\hline \multicolumn{5}{|c|}{ Phenological } \\
\hline DF & 70 & $10.80-81.21$ & 7 & $16.63-61.23$ \\
\hline $\mathrm{DM}$ & 150 & 13.44-91.55 & 15 & $15.12-56.34$ \\
\hline \multicolumn{5}{|c|}{ Yield related } \\
\hline 100SDW & 7 & $11.86-22.46$ & 55 & $11.23-80.55$ \\
\hline $\mathrm{BM}$ & 86 & $10.63-35.47$ & 44 & $11.54-63.51$ \\
\hline HI & 63 & $11.02-54.28$ & 41 & $16.19-81.58$ \\
\hline POD & 13 & $12.12-22.80$ & 4 & $27.77-59.30$ \\
\hline SPD & 43 & $10.98-35.91$ & - & - \\
\hline YLD & 82 & $10.04-54.36$ & 34 & $20.50-92.19$ \\
\hline \multicolumn{5}{|c|}{ Transpiration related } \\
\hline$\delta^{13} \mathrm{C}$ & 2 & $16.89-43.10$ & - & - \\
\hline \multicolumn{5}{|c|}{ Drought indices } \\
\hline DSI & 19 & $11.61-28.63$ & 3 & $70.09-80.95$ \\
\hline DTI & 26 & $15.64-41.28$ & 16 & $15.04-91.83$ \\
\hline Total & 686 & & 287 & \\
\hline
\end{tabular}

'TA103II-TA122' on CaLG01 was stable at two locations (PAT and HIR) (Table 3; ESM Table S8). In addition, 22 E-QTLs with up to $61.23 \%$ PVE were detected for both the traits (Table 4).

\section{Yield and yield-related traits}

The QTL analysis of six yield-related traits detected a total of 11 robust M-QTLs (3 for HI, 2 each for 100SDW, BM and YLD, 1 each for POD and SPD) which explained up to $58.20 \%$ PVE in ICCRIL03. For 100SDW, interestingly one QTL 'QR3100sdw03' flanked by 'NCPGR127NCPGR21' on CaLG04 was consistent across three seasons (2006, 2008 and 2009) and stable across all the five locations (PAT, HIR, SEH, NDL and DUG). Further, for POD QTL 'QR3pod01' flanked by 'NCPGR127-NCPGR21' on
CaLG04 was consistent across four seasons (2005, 2006, 2007 and 2009) (Table 3; ESM Table 8). Further in terms of E-QTLs, 294 robust E-QTLs explained up to 54.37 \% PVE (for 6 traits) in the case of ICCRIL03. Among 294 robust E-QTLs detected, one locus, namely 'TAA170', showed interaction in 21 of 82 robust E-QTLs for yield (ESM Table 11).

In the case of ICCRIL04, seven robust M-QTLs were detected (3 for YLD, 2 for $\mathrm{HI}$ and 1 for 100SDW and POD); of these QTL for 100SDW ' $Q R 4100 s d w 02$ ' flanked by 'CaM2093-ICCM0249' on CaLG04 was consistent across three seasons (2005, 2006 and 2007) and one QTL for POD 'QR4pod02' flanked by 'CaM0772TS45' on CaLG08 was consistent across two seasons (2006 and 2007). A total of 178 robust E-QTLs were identified (Table 4). 


\section{Transpiration efficiency}

No robust M-QTL for $\delta^{13} \mathrm{C}$ was detected in the case of ICCRIL03, while in the case of ICCRIL04, $\delta^{13} \mathrm{C}$ was not measured. Only two robust E-QTLs with 16.89 to $43.10 \%$ PVE were identified in ICCRIL03 (Table 4). This indicates that minor QTLs, showing interaction, play a significant role for TE.

\section{Drought tolerance and susceptible indices}

In case of ICCRIL03, one robust M-QTL with $11.23 \%$ PVE and 45 robust E-QTLs with up to $41.28 \%$ PVE were identified for DTI (Tables 3, 4). Among robust 45 E-QTLs, one locus, namely 'ICCM0257', was interacting among 7 QTLs identified in 2009 (ESM Table 11). In the case of ICCRIL04, two robust M-QTLs with 11.27-12.12\% PVE and 19 E-QTLs with up to $91.83 \%$ PVE were identified (Tables 3, 4). No consistent and stable QTLs were observed for DTI and DSI drought tolerance indices in both RIL populations.

\section{Consensus genetic and QTL map}

While comparing two intra-specific genetic maps, 43 marker loci were found common between two maps. These markers were considered as anchor markers and used for merging the genetic maps for construction of consensus genetic map. The consensus map comprised 352 loci and covered a total map distance of $771.39 \mathrm{cM}$. The length of LGs ranged from $58.44 \mathrm{cM}$ (CaLG05) to $155.99 \mathrm{cM}$ (CaLG07) (Table 2; Fig. 1). The density of markers on the map ranged from $1.11 \mathrm{cM} /$ marker on CaLG3 to $3.63 \mathrm{cM} /$ marker on CaLG07, with an average density of $2.30 \mathrm{cM} /$ marker. However, $6.36 \%$ (26) markers could not be integrated on to the consensus genetic map. Among different types of marker (SSR/STMS, EST-SSR, conserved intron spanning regions, CISR; cleaved amplified polymorphic sequence (CAPS) and DArT) loci, the consensus genetic map predominantly consists of SSR marker loci (322). Majority of the DArT loci $(40 \%)$ were confined to CaLG01, while the remaining DArT loci were mapped on CaLG07 and CaLG04. Of five CAPS markers used for mapping, only one marker (Tp684964) was mapped on CaLG04.

Detailed comparison using CMap among consensus map and population specific/component genetic maps revealed a very high congruency in terms of marker orders corresponding LGs and markers grouping on LGs (correlation coefficients varying from 0.86 to 0.99 ; Table 2 ). An example of correlation in one linkage group (CaLG04) has been shown in Fig. 2. The CaLG04 has seven markers common between two component genetic maps and six of these seven markers were placed on the consensus map. The figure shows a good conservation of marker order amongst consensus and the two genetic maps. Comparison of each LG across three maps can be visualized at http://cmap.icris at.ac.in/cmap/sm/cp/varshney/.

Efforts were also made to place the detected robust M-QTLs as mentioned earlier on the consensus map. All 25 and 20 robust M-QTLs detected in ICCRIL03 and ICCRIL04, respectively, were placed on the consensus map. These 45 robust M-QTLs for 14 traits and DTI contribute up to $58.20 \%$ PVE.

Genomic regions containing QTLs for several traits are much valued by breeders. In this context, we analyzed the detected QTLs and considered QTL cluster/co-localized QTLs if they represent for more than three traits. In case of ICCRIL03, two QTL clusters (each one on CaLG04 and CaLG08) were identified. A QTL cluster on CaLG04 colocalized 12 QTLs influencing 12 traits (RLD, RTR, SDW, DF, DM, SPD, PHT, POD, HI, YLD, BM and 100SDW) with up to $58.20 \%$ PVE (Fig. 1). Similarly, a cluster on CaLG08 clustered four QTLs influencing four traits (DF, DM, BM and PHT) with up to $26.87 \%$ PVE.

In the case of ICCRIL04, QTLs were co-localized on CaLG08. A total of six QTLs for six traits (DF, DM, PHT, POD, HI and PWD) with up to $31.32 \%$ PVE were co-localized between GA6 and NCPGR138 markers on CaLG08 (ESM Fig. 2). Furthermore, mapping of QTLs identified in two RIL populations on the consensus genetic map provided nine QTL clusters (Fig. 1). Among nine QTL clusters, QTL Cluster 1, QTL Cluster 2 and QTL Cluster 3 were located on CaLG01; QTL Cluster 4 on CaLG03, QTL Cluster 5 on CaLG04; QTL Cluster 6 on CaLG05; QTL Cluster 7 and QTL Cluster 8 were on CaLG06 and QTL Cluster 9 was on CaLG08.

\section{"QTL-hotspot" region for drought tolerance}

While analyzing robust M-QTLs in detail, an interesting genomic region $(29 \mathrm{cM})$ containing seven markers (ICCM0249, NCPGR127, TAA170, NCPGR21, TR11, GA24 and STMS11) was identified on CaLG04 of genetic map for ICCRIL03. This region contained 12 out of 25 (48 \%) robust M-QTLs for 12 traits (100SDW, RLD, DF, DM, BM, PHT, POD, HI, RTR, SDW, SPD and YLD). Furthermore, one consistent QTL each for SDW, PHT, POD and 100SDW and one stable QTL each for PHT and 100SDW were located in this genomic region (Table 3).

Similarly, one genomic region in the case of ICCRIL04, spanning $15 \mathrm{cM}$ with six markers (CaM2093, ICCM0249, TA130, CaM1214, NCPGR142 and TAA170) was identified on CaLG04 of the genetic map of ICCRIL04. However, only one consistent QTL was observed in this genomic region and none of the identified QTLs were stable. 


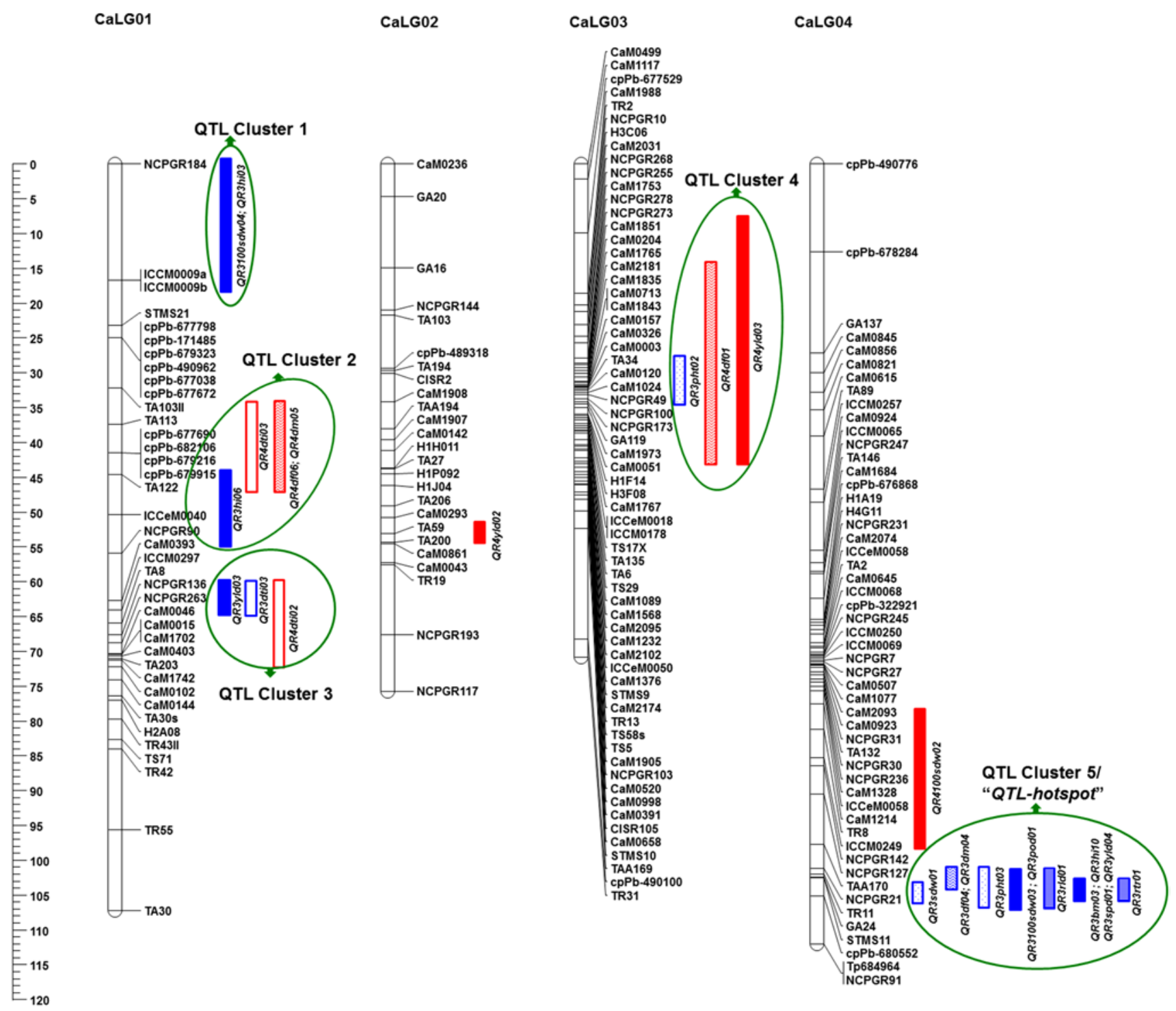

Fig. 1 Consensus genetic and QTL map comprising 352 marker loci based on two intra-specific mapping populations. Markers are shown on the right side of the LG, while map distances are shown on the left

While comparing genomic regions on CaLG04 of component genetic maps of two populations, two markers (ICCM0249 and TAA170) were found common in the regions. Therefore, the regions identified in two-component genetic maps are the same region in the chickpea genome. This region is the QTL Cluster 5. As this region contained a total of 13 robust QTLs for 12 traits with $58.20 \%$ PVE and identified in genetic maps of both RILs, we have designated this region as "QTL-hotspot" region in chickpea genome. As this "QTL-hotspot" contained four consistent and two stable QTLs for 12 traits and DTI with up to $58.20 \%$ PVE in ICCRIL03 and one consistent QTL with up to $26.68 \%$ PVE in ICCRIL04, this region can be considered as a side. The QTLs identified from the ICCRIL03 and ICCRIL04 populations are differentiated by different colors

promising drought tolerance candidate genomic region for molecular breeding.

\section{Discussion}

Towards understanding complexity of drought tolerance in chickpea, a few expression and functional genomics (Varshney et al. 2009; Deokar et al. 2011) and physiological (Zaman-Allah et al. 2011) studies were conducted in recent past; however, the genetics and molecular mechanisms for drought tolerance is still not well understood. This study reports genetics-based dissection of drought tolerance after 

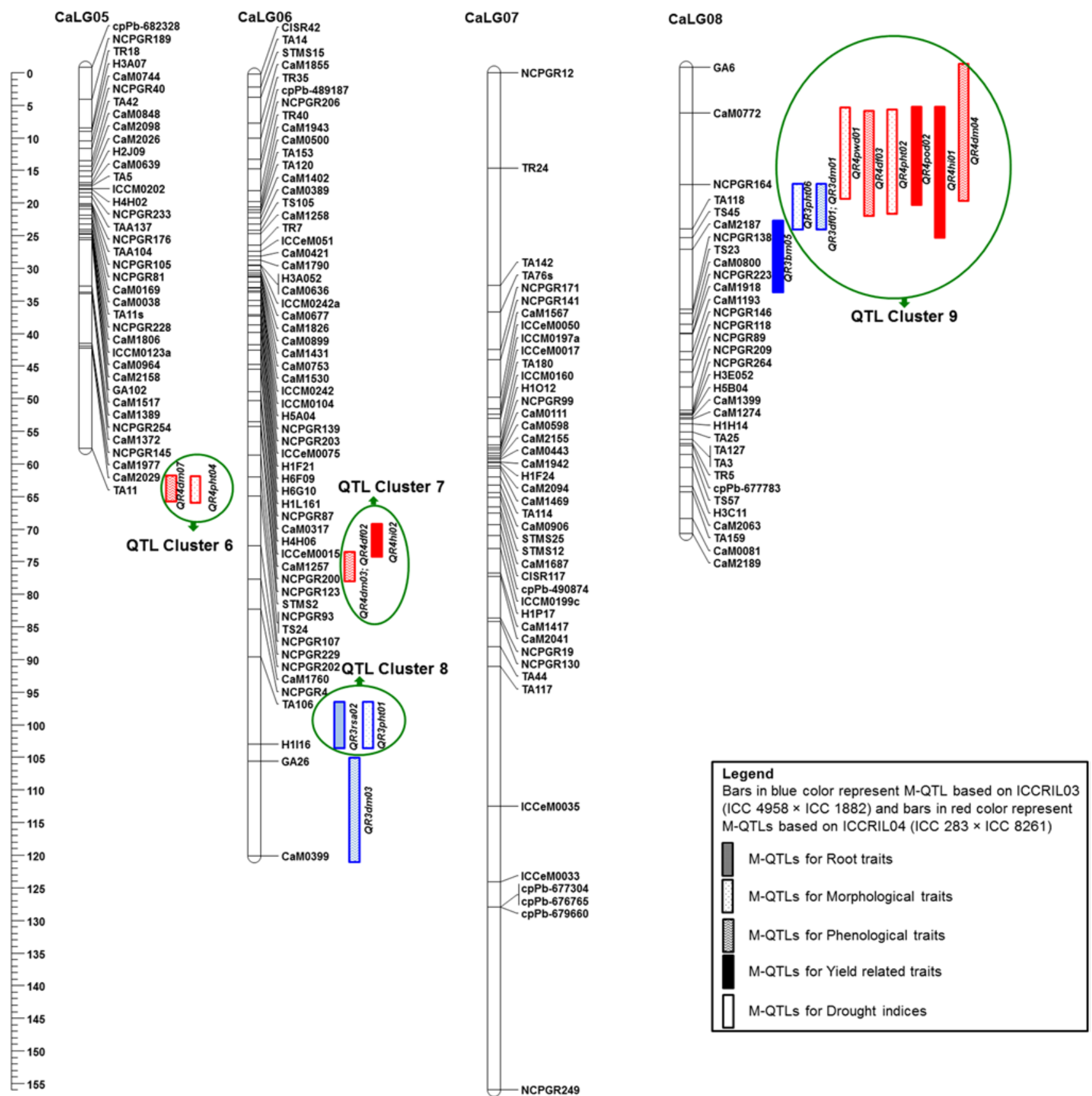

Fig. 1 continued

generating and analyzing extensive phenotyping and genotyping data on two segregating populations.

Extensive and precise phenotyping for drought tolerance

To better understand drought tolerance mechanism in chickpea, 20 drought tolerance component traits were phenotyped under 1-7 seasons at 1-5 locations in India. Detailed analysis of phenotyping data on six root traits indicated that the phenotypic variation among RILs in the ICCRIL03 population was almost double for all root traits studied compared to earlier studies (Serraj et al. 2004; Kashiwagi et al. 2008), although earlier studies deployed germplasm and RILs were studied in the present study. In the case of ICCRIL04, although variation among RILs was high, the variation between parental genotypes was comparatively low than that of the ICCRIL03. The broad sense heritability $\left(H^{2}\right)$ for the six root traits ranged from 0.07 


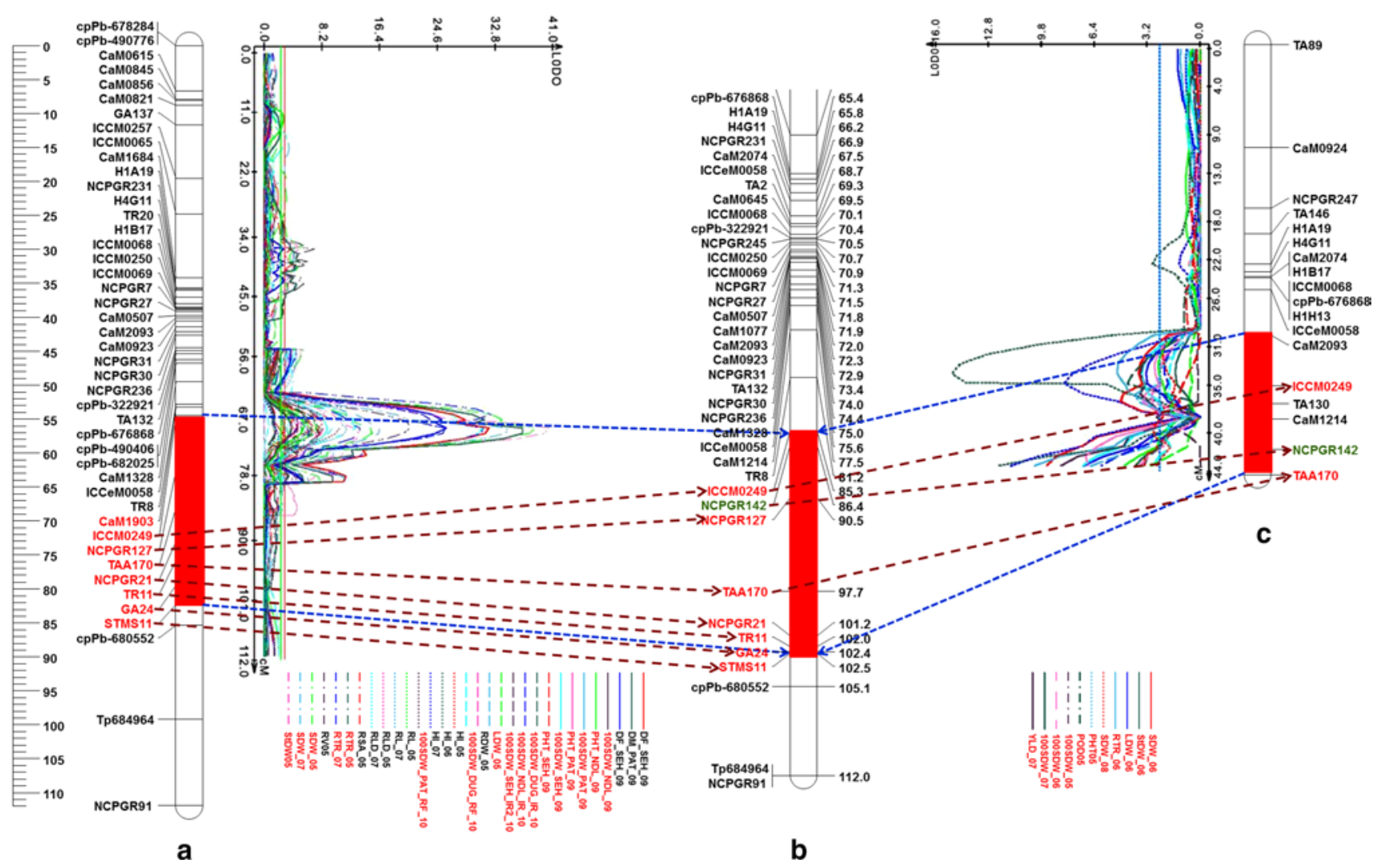

Fig. 2 Comparison of "QTL-hotspot" genomic region harboring QTLs for various drought tolerance-related traits identified on CaLG04 of two intra-specific mapping populations with genomic region on consensus map. a QTLs identified based on ICCRIL03

to 0.61 in the ICCRIL03 and $0.15-0.48$ in ICCRIL04. As reported earlier (Kashiwagi et al. 2005), the $H^{2}$ was high in case of RLD. Since RLD is associated with greater yield under terminal drought conditions, selection for such a trait with high $H^{2}$ in breeding may help enhancing the genetic gains and yield improvement in chickpea. The phenological traits such as DF and DM possessed high $H^{2}$ across locations and in different environments/seasons, indicating that selection for these traits will also be effective in breeding. Among yield-related traits, the high $H^{2}$ was observed in case of 100SDW across locations, seasons/environments indicating that 100SDW is the least affected trait by the environment and selection for this trait may positively improve yield under terminal drought conditions.

Genetic and consensus maps

Most of the dense genetic maps developed to date in chickpea are based on inter-specific crosses (Nayak et al. 2010; Thudi et al. 2011; Gaur et al. 2012; Hiremath et al. 2012). Although intra-specific genetic maps (Radhika et al. 2007; Gaur et al. 2011) as well as consensus maps based on
(ICC $4958 \times$ ICC 1882) mapping population. b CaLG04 of consensus genetic map. c QTLs identified based on ICCRIL04 (ICC $283 \times$ ICC 8261) mapping population. QTLs common to traits in both mapping populations are highlighted in red

intra-specific crosses were developed in chickpea (Radhika et al. 2007; Millàn et al. 2010; Gaur et al. 2011), marker density was very low and maximum number of marker loci (including random amplified polymorphic DNA, RAPD and sequence tagged microsatellites, STMS) mapped on to a single intra-specific genetic map are 138 (Gaur et al. 2011) and the consensus map has 229 markers (Millàn et al. 2010). The present study reports a significant improvement of marker density in the intra-specific component (2-fold) and consensus (1.5-fold) genetic maps. Consensus map reported here comprised 352 marker loci across all 8 LGs, spanning a total distance of $771.39 \mathrm{cM}$ and is developed based on two intra-specific RIL populations. Unlike other published maps for intra-specific mapping populations which contained anonymous markers (like RAPD, AFLP), the consensus map developed in the present study comprised mainly SSR markers. Marker order and marker distribution on individual genetic maps as well as consensus map were highly conserved $(P \leq 0.98$; Table 2$)$. Furthermore, comparison of the consensus map of the present study with the inter-specific map developed by Thudi et al. (2011) also revealed a high conservation of marker order 
and 38 markers were common between these two genetic maps. Sparse distribution of marker loci towards telomeres in the cases of CaLG01, CaLG02 and CaLG07 may be due to lower recombination rates and such kind of low marker densities in telomeric regions was also observed in earlier studies (Nayak et al. 2010; Thudi et al. 2011). Similarly, higher genomic SSR marker density towards the centromeres indicates the unequal recombination rates among the chickpea chromosomes.

High congruency in terms of marker order observed in case of component genetic maps and consensus map in the present study will be quite useful for ordering future genetic maps. Higher marker density of the consensus map, compared to other published maps (Millàn et al. 2010), will allow selection of specific markers for molecular breeding applications such as fine mapping, the development of novel genetic stocks (e.g., near isogenic lines and inbred backcross lines). This consensus map will also provide opportunities of anchoring with the physical map and facilitate mapping of known genes from legumes based on synteny.

\section{Simplification of complex traits}

In the present study, a large number of QTLs for several drought component traits have been identified by CIM analysis. Although QTL Cartographer, QTLNetwork and GMM program were used for detailed analysis, M-QTLs identified by QTL Cartographer and E-QTLs (3 loci interactions) identified by GMM program have been considered for further analysis. In order to gain deeper insights into drought tolerance, five groups of drought tolerance-related traits, namely root traits, morphological traits, phenological traits, yield and yield-related traits and TE, were attempted for genetic and molecular dissection (Ravi et al. 2011).

For six root traits analyzed in two RIL populations, a total of 18 M-QTLs were identified on all LGs except CaLG02. While considering only robust QTLs, 3 M-QTLs one each for RLD (CaLG04), RSA (CaLG06) and RTR (CaLG04) were found specific to only ICCRIL03. As ICCRIL03 and ICCRIL04 have ICC 4958 and ICC 8261 drought tolerant parents, ICC 4958 seems to have majoreffect QTLs for identified root traits. ICC 8261 either has only small-effect QTLs or robust QTLs present in the ICC 8261 could not be identified in this study. On the other hand, all identified 81 E-QTLs were robust and present in both RIL populations that indicate that epistatic interaction plays a significant role in expression of root traits.

In case of morphological traits, a total of 23 M-QTLs were found on all LGs except CaLG02. These QTLs included eight robust M-QTLs on five LGs for three traits, namely SDW (CaLG04), PHT (CaLG03, CaLG04, CaLG05, CaLG06 and CaLG08) and PWD (CaLG08).
QTLs for SDW and PWD were specific to populations. However, both populations share at least one QTL for PHT on CaLG08 that contribute $14.73 \%$ PVE (ICCRIL03) to $31.32 \%$ PVE (ICCRIL04). In addition, 112 E-QTLs were robust QTLs with up to $76.54 \%$ PVE on all LGs indicating prominent role of epistatic interaction in expression of morphological traits.

For phenological traits, a total of 23 M-QTLs on all LGs except CaLG02 including 13 robust M-QTLs were detected in two RIL populations for both traits (DF and DM). Occurrence of 13 robust M-QTLs on 6 LGs indicates quantitative nature of the traits. Furthermore, identification of 242 E-QTLs present all over the genome highlights the involvement of epistatic interaction for phenological traits.

Yield is considered to be important trait for chickpea farmers in semi-arid regions where terminal drought is prevailing. A total of $51 \mathrm{M}$-QTLs and $480 \mathrm{E}-\mathrm{QTL}$ were identified for 6 yield and yield-related traits in two RIL populations. However, only $18 \mathrm{M}-\mathrm{QTLs}$ present on all LGs except CaLG05 and CaLG07, and 472 E-QTLs present on all LGs were robust. As all $18 \mathrm{M}$-QTLs are specific to one of two populations and contribute a range of phenotypic variation, yield and yield-related traits show the complex nature of genetics.

For TE-related traits, a total of 2 M-QTLs (both on CaLG04) and 2 E-QTLs were found in the ICCRIL03 of which only E-QTLs were robust. Low number of QTLs identified in the study is a function of use of smaller set of phenotyping data obtained in only one population and in 1 year as compared to datasets for other traits.

\section{Candidate genomic regions for molecular breeding}

In any breeding program, the traits to be considered as potential selection targets for improving yield under waterlimited conditions must be genetically correlated with yield, and should have a greater $H^{2}$ than yield itself (Blum 2011). As mentioned earlier, root traits are drought avoidance traits, phenological traits (DF and DM) are drought escape traits and WUE or TE is drought tolerance traits. Improving any one or combination of these traits will improve yield under drought conditions (Gaur et al. 2008). Of course, yield and yield-related traits like HI under drought conditions are the ultimate targets in a breeding program (Krishnamurthy et al. 2013b).

The present study reports nine QTL clusters that have robust QTLs for all of the above-mentioned traits except TE. For instance, QTL Cluster 5 contains QTLs for root traits (RLD $10.90 \%$ PVE, RTR $16.67 \%$ PVE), phenological traits (DF $24.49 \%$ PVE, DM $19.71 \%$ PVE) as well as yield and yield-related traits (100SDW 58.20\% PVE, POD $23.18 \%$, BM $21.32 \%$, SPD $42.07 \%$, HI $11.69 \%$ ). In fact, this region has been referred as "QTL-hotspot" as this 
region contained several stable and consistent QTLs with higher PVE. While considering all the QTLs, this region contains 17 (22.07\% of total) QTLs for 15 traits including TE and DTI were identified in ICCRIL03 that contributes from 4.49 to $58.2 \%$ PVE. Of these QTLs, four were consistent and two were stable QTLs. Furthermore, seven QTLs for five traits identified in the ICCRIL04 also fell in the same region. In brief, this region has 22 QTLs for 15 traits for all the five groups of traits analyzed across two RILs. Therefore, this region seems to be of utmost importance for introgression in elite varieties for enhancing yield under drought conditions.

In addition to above, QTL Cluster 9 present on CaLG08 also seems to be an interesting genomic region for targeting for molecular breeding as it contains QTLs for DF (26.87 \%), DM (18.83\%), HI (14.04\%), PHT (31.32\%), PWD (15.84 \%) and POD (14.38 \%). Hence, introgression of this cluster will not only improve the component traits and but also yield in chickpea under drought as it improves HI, a key component trait for estimating yield under drought (Passioura 1977). Introgression of QTL Cluster 1, QTL Cluster 5, QTL Cluster 7 and QTL Cluster 8 will improve $\mathrm{HI}$ in total. Larger RSA will enhance soil contact and enable absorption of more available water, thus avoiding drought. Introgression of QTL Cluster 7 on CaLG06 simultaneously improves both drought escape traits like DM and drought avoidance traits like RSA.

Furthermore, large number of epistatic QTLs for different traits identified in present study indicates that the QTLs with minor effects/no effect interact with the other loci and influence the expression of the traits. For instance, although no robust QTL was identified for RLD, an important drought avoidance trait, in the genetic background of ICCRIL04, nine epistatic interactions were identified with up to $44.21 \%$ PVE. Nevertheless, epistatic interactions with high phenotypic variation were identified for the traits like RDW, RV and RDp, although no robust QTLs were identified across any genetic background. Further, for $\delta^{13} \mathrm{C}$ an indirect measure of TE, which in turn is an important trait for estimating yield under drought conditions (Krishnamurthy et al. 2013a), no robust QTL was detected; however, epistatic interactions with up to $43.10 \%$ PVE were identified in the case of ICCRIL03. Therefore, for harnessing such epistatic interactions, genomic selection (GS) will be the best alternative in achieving larger genetic gains in shorter periods (Varshney et al. 2012).

\section{Conclusion}

The present study reports on the development of two intraspecific genetic maps of chickpea that were integrated into a single consensus map containing 352 markers, with an average marker density of $2.3 \mathrm{cM} /$ marker, increasing dramatically the density over previously published genetic maps. The consensus map with QTLs integrated will be a valuable resource that will prompt the chickpea research community for next generation genomic and genetic studies. This study also provides nine QTL clusters containing QTLs for all target traits-drought avoidance, drought escape and drought tolerance. Among these QTL clusters, the QTL Cluster 5 on CaLG04, referred as " $Q T L$ hotspot" harboring stable and consistent QTLs for several drought tolerance traits, is the most significant region in molecular breeding for improving yield under terminal drought conditions (Varshney et al. 2013). In addition, there are several other QTL clusters that either individually or in combination can be target for introgressing or pyramiding superior alleles for drought tolerance in elite varieties. Analysis of QTL map with genome sequence has suggested the length of the "QTL-hotspot" as $7.74 \mathrm{Mb}$ region in the genome. This region contains few hundred genes. Availability of high-throughput sequencing technologies offers the possibility to fine map and eventually clone the QTL region, e.g., "QTL-hotspot", and identify the candidate genes or/and transcription factors to understand the molecular mechanisms for drought tolerance.

Acknowledgments Technical assistance from Srivani Gudipati, Veerender Peddinti and Abdul Gafoor are greatly appreciated. Special thanks are due to Trushar Shah and A. BhanuPrakash for their contribution in uploading the mapping data to CMap server. The work was supported by Tropical Legumes I (TLI) project funded by Bill and Melinda Gates Foundation (BMGF) through CGIAR Generation Challenge Program (GCP; http://www.generationcp.org/). This work has been undertaken as part of the CGIAR Research Program on Grain Legumes. ICRISAT is a member of CGIAR Consortium.

Conflict of interest The authors declare that they have no conflict of interest.

Open Access This article is distributed under the terms of the Creative Commons Attribution License which permits any use, distribution, and reproduction in any medium, provided the original author(s) and the source are credited.

\section{Supplementary Figures on CMap site}

The CMap site http://cmap.icrisat.ac.in/cmap/sm/ $\mathrm{cp} /$ varshney/ presents following, in addition to supplementary material of this article (ESM Table S1 to ESM Table S12 and ESM Figure S1 to ESM Figure S2): i) Consensus genetic map with 352 loci, ii) Correspondence of different linkage groups (LG1 to LG8) of Thudi et al. (2011) and two intra-specific maps (ICC $4958 \times$ ICC 1882 and ICC $283 \times$ ICC 8261 presented in this article). 


\section{References}

Abate T, Alene AD, Bergvinson D, Shiferaw B, Silim S, Orr A, Asfaw S (2012) Tropical grain legumes in Africa and south Asia: knowledge and opportunities. SAT eJournal 10:1-12. http://ejournal.icrisat.org/ Volume10/IMPI/Research_development.pdf

Ahmad F, Gaur PM, Croser J (2005) Chickpea (Cicer arietinum L.). In: Singh RJ, Jauhar PP (eds) Genetic resources, chromosome engineering, and crop improvement-Grain Legumes, vol 1. CRC Press, Boca Raton, pp 187-217

Blum A (2011) Plant breeding for water-limited environments, vol 14. Springer, New York, p 258

Bohra A, Saxena RK, Gnanesh BN, Saxena KB, Byregowda M, Rathore A, KaviKishor PB, Cook D, Varshney RK (2012) An intra-specific consensus genetic map of pigeonpea [Cajanus cajan (L) Millspaugh] derived from six mapping populations. Theor Appl Genet 125:1325-1338

Cuc LM, Mace E, Crouch J, Quang VD, Long TD, Varshney RK (2008) Isolation and characterization of novel microsatellite markers and their application for diversity assessment in cultivated groundnut (Arachis hypogaea). BMC Plant Biol 8:55

Deokar AA, Kondawar V, Jain PK, Karuppayil M, Raju NL, Vadez V, Varshney RK, Srinivasan R (2011) Comparative analysis of expressed sequence tags (ESTs) between drought-tolerant and -susceptible genotypes of chickpea under terminal drought stress. BMC Plant Biol 11:70

FAOSTAT (2011) http://faostat3.fao.org/home/index.html

Gaur PM, Krishnamurthy L, Kashiwagi J (2008) Improving drought avoidance traits in chickpea (Cicer arietinum): current status of research at ICRISAT. Plant Prod Sci 11:3-11

Gaur R, Sethy NK, Choudhary S, Shokeen B, Gupta V, Bhatia S (2011) Advancing the STMS genomic resources for defining new locations on the intra-specific genetic linkage map of chickpea (Cicer arietinum L.). BMC Genomics 12:117

Gaur R, Azam S, Jeena G, Choudhary S, Jain M, Yadav G, Tyagi AK, Chattopadhyay D, Bhatia S (2012) High-throughput SNP discovery and genotyping for constructing a saturated linkage map of chickpea (Cicer arietinum L.). DNA Res. doi:10.1093/dnares/ dss018

Gautami B, Pandey MK, Vadez V, Nigam SN, Ratnakumar P, Krishnamurthy L, Radhakrishnan T, Gowda MVC, Narasu ML, Hoisington DA, Knapp SJ, Varshney RK (2012) QTL analysis and consensus genetic map for drought tolerance traits based on three RIL populations of cultivated groundnut (Arachis hypogaea L.). Mol Breed 32:757-772

Golabadi M, Arzani A, Mirmohammadi Maibody SAM (2006) Assessment of drought tolerance in segregating populations in durum wheat. Afr J Agric Res 1:162-171

Gujaria N, Kumar A, Dauthal P et al (2011) Development and use of genic molecular markers (GMMs) for construction of a transcript map of chickpea (Cicer arietinum L.). Theor Appl Genet 122:1577-1589

Hiremath PJ, Kumar A, Penmetsa RV et al (2012) Large-scale development of cost-effective SNP marker assays for diversity assessment and genetic mapping in chickpea and comparative mapping in legumes. Plant Biotechnol J 10:716-732

IPCC (2007) IPCC Fourth Assessment Report. Climate Change, Geneva

Kashiwagi J, Krishnamurthy L, Upadhyaya HD, Krishna H, Chandra S, Vadez V, Serraj R (2005) Genetic variability of drought-avoidance root traits in the mini-core germplasm collection of chickpea (Cicer arietinum L.). Euphytica 146:213-222

Kashiwagi J, Krishnamurthy L, Crouch JH, Serraj R (2006) Variability of root length density and its contributions to seed yield in chickpea (Cicer arietinum L.) under terminal drought stress. Field Crops Res 95:171-181
Kashiwagi J, Krishnamurthy L, Serraj R, Crouch JH, Panwar S (2007) Rapid and reliable screening technique for root traits in chickpea. J Food Legumes 20:67-70

Kashiwagi J, Krishnamurthy L, Upadhyaya HD, Gaur PM (2008) Rapid screening technique for canopy temperature status and its relevance to drought tolerance improvement in chickpea. SAT eJournal 6:1-4. http://www.icrisat.org/journal/volume6/chickpea _pigeonpea/kashiwagil.pdf

Kashiwagi J, Krishnamurthy L, Gaur PM, Upadhyaya HD, Varshney RK, Tobita S (2013) Traits of relevance to improve yield under terminal drought stress in chickpea (C. arietinum L.). Field Crops Res 145:88-95

Krishnamurthy L, Kashiwagi J, Gaur PM, Upadhyaya HD, Vadez V (2010) Sources of tolerance to terminal drought in the chickpea (Cicer arietinum L.) minicore germplasm. Field Crops Res 119:322-330

Krishnamurthy L, Kashiwagi J, Tobita S, Ito O, Upadhyaya HD, Gowda CLL, Gaur PM, Sheshshayee MS, Singh S, Vadez V, Varshney RK (2013a) Variation in carbon isotope discrimination and its relationship with harvest index in the reference collection of chickpea germplasm. Funct Plant Biol 40:1350-1361

Krishnamurthy L, Kashiwagi J, Upadhyaya HD, Gowda CLL, Gaur PM, Singh S, Purushothaman R, Varshney RK (2013b) Partitioning coefficient $-\mathrm{a}$ trait that contributes to drought tolerance in chickpea. Field Crops Res 149:354-365

Lichtenzveig J, Scheuring C, Dodge J, Abbo S, Zhang HB (2005) Construction of BAC and BIBAC libraries and their applications for generation of SSR markers for genome analysis of chickpea, Cicer arietinum L. Theor Appl Genet 110:492-510

Millàn T, Winter P, Jüngling R, Gil J, Rubio J, Cho S, Cobos MJ, Iruela M, Rajesh PN, Tekeoglu M, Kahl G, Muehlbauer FJ (2010) A consensus genetic map of chickpea (Cicer arietinum L.) based on 10 mapping populations. Euphytica 175:175-189

Mir RR, Zaman-Allah M, Sreenivasulu N, Trethowan R, Varshney RK (2012) Integrated genomics, physiology and breeding approaches for improving drought tolerance in crops. Theor Appl Genet 125:625-645

Nayak SN, Zhu H, Varghese N et al (2010) Integration of novel SSR and gene-based SNP marker loci in the chickpea genetic map and establishment of new anchor points with Medicago truncatula genome. Theor Appl Genet 120:1415-1441

Passioura JB (1977) Grain yield, harvest index, and water use of wheat. J Aust Institute of Agril Sci 43:117-120

Radhika P, Gowda SJM, Kadoo NY, Mhase LB, Jamadagni BM, Sainani MN, Chandra S, Gupta VS (2007) Development of an integrated intraspecific map of chickpea (Cicer arietinum L.) using two recombinant inbred line populations. Theor Appl Genet 115:209-216

Ravi K, Vadez V, Isobe $S$ et al (2011) Identification of several small main-effect QTLs and a large number of epistatic QTLs for drought tolerance related traits in groundnut (Arachis hypogaea L.). Theor Appl Genet 122:1119-1132

Serraj R, Sinclair TR (2002) Osmolyte accumulation: can it really help increase crop yield under drought conditions? Plant Cell Environ 25:333-341

Serraj R, Krishnamurthy L, Kashiwagi J, Kumar J, Chandra S, Crouch JH (2004) Variation in root traits of chickpea (Cicer arietinum L.) grown under terminal drought. Field Crops Res 88:115-127

Sethy NK, Shokeen B, Edwards KJ, Bhatia S (2006) Development of microsatellite markers and analysis of intra-specific genetic variability in chickpea (Cicer arietinum L.). Theor Appl Genet 112:1416-1428

Thudi M, Bohra A, Nayak SN et al (2011) Novel SSR markers from BAC-end sequences, DArT arrays and a comprehensive genetic map with 1,291 marker loci for chickpea (Cicer arietinum L.). PLoS ONE 6(11):e27275 
Toorchi M, Shashidhar HE, Sharma N, Hittalmani S (2004) Tagging QTLs for maximum root length in rainfed lowland rice by combined selective genotyping and STMs markers. 4th International Crop Science Congress. http://cropscience.org.au/icsc2004/ poster/3/4/1/1413_toorchi.html

Tuberosa R (2012) Phenotyping for drought tolerance of crops in the genomics era. Front Physiol 3:347

Tuberosa R, Salvi S (2007) From QTLs to genes controlling root traits in maize. In: Spiertz JHJ, Struik PC, van Laar HH (eds) Scale and complexity in plant systems research: gene-plant-crop relations. Springer, The Netherlands, pp 15-24

Van Ooijen JW (2006) Join Map 4: software for the calculations of genetic linkage maps in experimental populations. Kyazma B. V, Wageningen

Varshney RK, Hiremath PJ, Lekha P, Kashiwagi J, Balaji J, Deokar AA, Vadez V, Xiao Y, Srinivasan R, Gaur PM, Siddique KHM, Town CD, Hoisington DA (2009) A comprehensive resource of drought-and salinity responsive ESTs for gene discovery and marker development in chickpea (Cicer arietinum L.). BMC Genomics 10:523

Varshney RK, Thudi M, May GD, Jackson SA (2010) Legume genomics and breeding. In: Janick J (ed) Plant breeding reviews, vol 33. Wiley, USA, pp 257-304

Varshney RK, Pazhamala L, Kashiwagi J, Gaur PM, Krishnamurthy L, Hoisington DA (2011) Genomics and physiological approaches for root trait breeding to improve drought tolerance in chickpea (Cicer arietinum L.). In: de Oliveira AD, Varshney RK (eds) Root genomics. Springer, Germany, pp 233-250

Varshney RK, Ribaut JM, Buckler ES, Tuberosa R, Rafalski JA, Langridge $P$ (2012) Can genomics boost productivity of orphan crops? Nat Biotechnol 30:1172-1176

Varshney RK, Gaur PM, Chamarthi SK, Krishnamurthy L, Tripathi S, Kashiwagi J, Samineni S, Singh VK, Thudi M, and Jaganathan D (2013) Fast-Track Introgression of "QTL-hotspot" for root traits and other drought tolerance traits in JG 11, an elite and leading variety of chickpea. Plant Genome. doi:10.3835/plantgen ome2013.07.0022

Wang HL, Yu DY, Wang YJ, Chen SY, Gai JY (2004) Mapping QTLs of soybean root weight with RIL population NJRIKY. Yi Chuan 26:333-336

Wang S, Basten CJ, Zeng ZB (2010) Windows QTL cartographer 2.5. Department of Statistics, North Carolina State University, Raleigh, NC. http://statgen.ncsu.edu/qtlcart/WQTLCart.htm

Winter P, Pfaff T, Udupa SM, Hüttel B, Sharma PC, Sahi S, ArrequinEspinoza R, Weigand F, Muehlbauer FJ, Kahl G (1999) Characterization and mapping of sequence-tagged microsatellite sites in the chickpea (C. arietinum L.). Mol Gen Genet 262:90-101

Zaman-Allah M, Jenkinson DM, Vadez V (2011) Chickpea genotypes contrasting for seed yield under terminal drought stress in the field differ for traits related to the control of water use. Funct Plant Biol 38:270-281 